\title{
An improved 2-point block backward differentiation formula for solving stiff initial value problems
}

\begin{abstract}
A new block method that generates two values simultaneously is developed for the integration of stiff initial value problems. The method is proven to be A $\ddot{i}$ stable and is a super class of the $2 \ddot{i}$ point block backward differentiation formula (BBDF). A comparison is made between the method, 1 point backward differentiation formula (BDF) and the 2 point BBDF methods. The numerical results indicate that the new method outperformed the 1 point BDF and the 2 point BBDF methods in terms of accuracy and stability. The total number of steps to complete the integration by the 1 point BDF method is reduced to half. Computation time for the method is also competitive.
\end{abstract}

Keyword: A-stability; Block method; Improved block backward differentiation formula; Initial value problems; Stiff 